\title{
Les migrations intra-balkaniques des années 1990 et 2000 à l'ombre des constructions nationales.
}

\author{
Guillaume Javourez, IDEMEC, UMR 7307 AMU-CNRS, Aix-en-Provence \\ Pierre Sintès, TELEMME, UMR 7303 AMU-CNRS, Aix-en-Provence
}

Durant les années 1990 et 2000, la Grèce devient une destination majeure pour des migrants venus des pays balkaniques, au premier rang desquels de nombreux albanais. Passant du statut de pays d'émigration à celui de pays d'immigration et signant les accords de Schengen, la Grèce a fait évoluer son arsenal législatif en conséquence. Entre règlements communautaires et particularités nationales, la construction du dispositif de contrôle des frontières du nord de ce pays illustre la concomitance de dynamiques continentales et d'autres influencées sur le long cours par des représentations en liens avec les processus de construction nationale dans la région.

In the 1990's, Greece became an important destination for migrants from other Balkan countries, and mostly from Albania. Moving from emigration to immigration country, Greece had to adapt its legal framework regarding this new context. Between EU community regulations and national particularities, the building of a dispositive of border control in Northern Greece illustrates the coexistence of continental dynamics and others influenced by social representations connected to the long term nation-building processes prevailing in this area.

Depuis 2015, l'actualité migratoire des pays européens a conduit à l'intensification des débats sur la place de l'échelon national dans le contrôle frontalier. Qu'il s'agisse de questions sécuritaires ou migratoires (voire des deux), l'hypothèse d'une perte de souveraineté de l'État sur ses frontières est omniprésente dans les discours, en particulier pour les forces politiques qui promeuvent une vision strictement statonationale de la société comme du territoire. Il est vrai que, depuis les années 1990 et la signature des accords de Schengen, l'Union européenne semble être devenue un acteur majeur de la gestion des frontières des États-membres, tant par son rôle normatif que par son implication concrète dans de nombreuses actions de terrain, tel qu'elles peuvent être observées dans les pays de l'Europe du Sud-est (Javourez, Pillant, Sintès, 2017). Dans le même temps, la politique d'élargissement de l'Union et l'intégration de l'acquis Schengen dans l'acquis communautaire étendait l'impact des politiques migratoires européennes aux pays candidats, parmi lesquels notamment les pays dits des «Balkans occidentaux ».

Les règles du franchissement frontalier dans cette région sont donc théoriquement soumises à la réglementation européenne autour de procédures standardisées à l'échelle de l'Union, reléguant à l'arrière-plan un échelon national sollicité uniquement dans le cadre de l'application de ces règlements. Dans un tel contexte, l'étude des processus migratoires balkaniques des années 1990 à 2000 permet d'observer de façon privilégiée la mise en place progressive de ces politiques communautaires et la redistribution des rôles entre institutions européennes et États. Par l'observation des dynamiques migratoires et de leurs impacts politiques et sociaux, cette période conduit également à interroger la permanence d'un échelon décisionnel, et aussi et surtout d'un échelon d'analyse et de compréhension, qui serait proprement national quand il s'agit d'aborder, par le terrain, la réalité des migrations ou des circulations dans l'espace 
européen. L'évocation des « constructions nationales » dans le titre de cet article renvoie à l'idée que des particularités issues de ces processus idéologiques, sociaux et territoriaux de long terme, qui touchent les Balkans mais aussi l'ensemble de l'Europe depuis plusieurs siècles, seraient les matrices d'un ensemble de valeurs et de représentations qui influencent les modalités du contrôle frontalier tel qu'ils ont été mis en place par les États. Les pays de la péninsule balkanique constituent alors un terrain particulièrement favorable pour un tel sujet. Loin de vouloir renforcer la vision d'un particularisme balkanique, l'observation de cette région se justifie plutôt par le fait qu'elle demeure une marge pour l'UE, un « front d'intégration » qui rendrait plus visible un décalage permanent entre la mise en œuvre des processus communautaires à l'échelle du continent et leur applications (ou leur interprétations) locales. Les Balkans ne sont pas à cet égard un cas particulier, les spécificités de la région permettant au contraire de rendre compte, avec plus de clarté, des processus communs à l'ensemble européen.

Pour aborder une telle question, nous nous intéresserons à la situation de la Grèce des années 1990 et 2000, alors que le pays était touché essentiellement par des flux migratoires intra-balkaniques. Nous analyserons plus particulièrement les régimes de franchissement frontalier tels qu'ils ont été mis en œuvre à cette période par la République Hellénique à partir de travaux de terrain conduits à ses frontières avec l'Albanie et la Macédoine du Nord'1.

\section{Les Balkans en transit (1990-2000)}

Les années 1990 et 2000 ont marqué le retour des mobilités et des migrations pour les pays des Balkans. Il faut en effet remonter assez loin dans le temps, au 19e et début du $20^{\text {e }}$ siècles, pour retrouver une telle mobilité des populations au sein de la région, en raison, en particulier, des conflits qui s'y déroulent jusqu'à la seconde guerre mondiale ${ }^{2}$. Après cette date, les Balkans connaissent, une sorte de mise en retrait des systèmes de mobilités internationales, mises à part les migrations de travailleurs grecs et yougoslaves vers les pays de l'Europe occidentale ou des destinations plus lointaines (Australie ou Etats-Unis).

La période suivante contraste beaucoup avec ce temps de pause. En effet, depuis 1990, l'ensemble de la péninsule connaît un regain de mobilités transfrontalières et de migrations suite aux bouleversements géopolitiques qui s'y produisent, à l'image de ce qui se passe dans le reste de l'Europe orientale (Morokvasic, 1992 ; de Tinguy, 2000 ; Whitol de Wenden 2001). A partir de cette date, les Balkans contribuent de manière soutenue au mouvement de diversification des origines, de multiplication des pays de départ et de destination qui caractérise à présent les migrations internationales. Ces flux migratoires, qui peuvent être identifiés sous la catégorie des "migrations de travail», affectent parfois massivement les pays balkaniques. En Albanie, le solde migratoire dans la décennie 1990 est négatif de 700.000 personnes pour un pays de 3 millions

\footnotetext{
${ }^{1}$ Le changement de nom de République de Macédoine en République de Macédoine du Nord a été acté par le parlement du pays en janvier 2019, mettant fin au conflit diplomatique qui l'opposait à la Grèce depuis 1991 à propos de cette dénomination. A propos de la crise macédonienne, voir Skoulariki, 2003.

${ }^{2}$ En 1949, la fin de la guerre civile grecque pouvant en être considérée comme le dernier acte pour ces très nombreuses mobilités forcées qui ont touché les populations des Balkans qui ont accompagnées la disparition de l'Empire ottoman.
} 
d'habitants, et on estime qu'1/5e de la population albanaise est partie en migration à l'étranger. On peut également mentionner la Roumanie et la Bulgarie comme pays de départ avec des contingents nombreux : on dénombrait au début des années 2000 plus de 700.000 émigrés pour une population de 8 millions de personnes en Bulgarie, et 2 millions pour 22 millions d'habitants en Roumanie. La décennie 1990 marque également le retour dans la région des "migrations forcées » survenues à l'occasion des conflits qui sévissent dans l'espace ex-yougoslave : plus de 900.000 personnes en sont les victimes entre 1992 et 2004. Dans ce contexte, notamment marqué par les nombreuses migrations en provenance de Bosnie-Herzégovine ${ }^{3}$, la Slovénie, la Croatie et la Serbie, deviennent des pays de réception indiscutables.

Dans ces derniers cas, il n'est pas étonnant de voir combien les flux migratoires forcés sont tout entier modelés par des logiques nationales et historiques. Mais, plus curieusement (ou en tous cas de manière plus discrète), des observations de terrain nous permettent de constater que les flux que l'on a qualifié plus haut d'« économiques » peuvent aussi être touchés par des logiques qui ne se conforment pas uniquement aux grandes tendances de l'actualité migratoire de l'époque, et qui répondent plutôt à des logiques nationales voire locales inscrites dans le temps long de cette région.

\section{La Grèce dans les années 1990 : nouvelle terre d'immigration}

Force est de constater que, pour la Grèce également, la chute des régimes bureaucratiques Est-européen au début des années 1990 a été un événement géopolitique majeur. Le pays n'était certes pas concerné par ce que l'on a appelé la «transition» économique et politique, mais celle-ci a eu pour conséquence de transformer pour la première fois de son histoire ce pays en une véritable terre d'accueil pour de très nombreux migrants venus principalement des pays alentours ${ }^{4}$. En effet, c'est durant cette période que des contingents importants de Géorgiens, de Roumains, de Bulgares et surtout d'Albanais s'installent en Grèce ; l'arrivée de ces migrants devenant pour ce pays l'un des phénomènes les plus marquants de son histoire récente.

Parmi eux, ce sont les Albanais qui sont les plus nombreux et les plus significatifs pour l'opinion publique grecque, car ils s'installent sur l'ensemble du territoire, de la moindre petite île aux bourgades reculées des montagnes, des lointaines périphéries urbaines aux centres des grandes villes. Pour faire face à cette situation, les autorités grecques se conforment pendant presque dix années au principe du... laissez-faire. Mis à part le premier décret de 1991, qui renforçait le contrôle frontalier et facilitait les expulsions, et avant les premières régularisations de 1997 et les réformes qui débutent en 2001, le dispositif en vigueur était toujours celui prescrit par la loi $n^{\circ} 4310$ de 1929 . Il a fallu attendre presqu'une décennie pour qu'un cadre légal adapté aux réalités migratoires contemporaines soit mis en place progressivement. Ce mouvement s'effectue en étroite relation avec les nécessités domestiques (économiques et politiques) mais aussi avec la situation internationale puisque le pays, signataire des accords de Schengen depuis 1992, devait se préparer à leur entrée en vigueur au tournant des années 20005. Les

\footnotetext{
${ }^{3}$ Le pays a, durant cette période, perdu 500.000 personnes sur une population de moins de cinq millions d'habitants

${ }^{4}$ Pour la première fois, si l'on fait exception des conséquences de l'échange de population faisant suite au traité de Lausanne de 1923 qui peuvent être vus comme le premier temps de formation de l'espace helladique au contact d'un flux massif d'immigrants (Parsanoglou, 2010).

${ }^{5}$ L'entrée en vigueur des accords de Schengen pour la Grèce date du 26 mars 2000.
} 
premières régularisations de 1997 semblent anticiper cet événement tout en permettant de ménager l'électorat rural qui voyait d'un mauvais œil la disparition dans les rafles policières de cette main d'œuvre essentielle à la bonne tenue de son activité.

C'est aussi l'ampleur prise par le "problème albanais » dans le courant des années $1990^{6}$ qui pourrait expliquer la diligence des gouvernants grecs à contrôler ainsi le groupe de migrants le plus nombreux sur son sol : c'est en effet à cette période que l'embrasement du Sud de l'Albanie à la suite de l'effondrement des systèmes financiers informels (1997) renforçait indirectement la généralisation de la migration familiale vers la Grèce. Des lois plus précises suivent, en 2001 et 2005, qui visent à lier les migrations aux besoins du marché du travail en créant notamment des agences de recrutement de travailleurs en Albanie et dans d'autres pays des Balkans. A cette volonté s'ajoute celle d'un contrôle ferme à la frontière avec le renforcement du corps des gardes-frontières et une série d'accords de réadmission avec les pays riverains de la Grèce, en vue d'éviter notamment que l'Albanie ne devienne la porte d'entrée du pays. Ils visaient aussi la Macédoine du Nord que la porosité des frontières avec la Grèce avait transformé en pays de transit notoire depuis l'Albanie.

\section{L'instauration du contrôle frontalier en Grèce, entre règlementations européens et dynamiques nationales}

L'application par la Grèce d'un nouveau cadre gestionnaire se voulait adaptée à l'évolution de la situation migratoire du pays, tout en convergeant avec ce qui se passait ailleurs en Europe dans le cadre d'une communautarisation croissante du contrôle frontalier. Il est pourtant possible d'y voir affleurer des considérations très éloignées d'un tel mouvement tant semblent également puissants les cadres produits par le processus de construction nationale grecque, ainsi que la marque laissée par l'épaisseur des relations historiques entre les Etats de la région. C'est le cas par exemple des modalités même de l'application des accords de Schengen qui ont été directement affectées par des questions de géopolitique régionale alors que la Grèce a longtemps exclu leur application aux citoyens de la Macédoine du Nord en raison du conflit diplomatique qui se jouait alors autour du nom de leur pays (Kondonis, 2005).

Le cas des migrants albanais est encore plus éloquent pour illustrer cette influence du temps long sur la réalité migratoire. En effet, ceux-ci pâtissent en Grèce d'un ensemble de représentations stéréotypées très négatives. Ces stéréotypes sont parfois articulés avec le fait que ces migrants sont les tenants, parfois malgré eux, d'une mémoire et d'une légitimité concurrente sur certaines zones appartenant aujourd'hui au territoire grec mais historiquement revendiquées par certains Albanais (la «Tchamerie », actuellement englobée dans la région grecques d'Épire, au nord-ouest du pays), ou sur des régions situées en Albanie mais revendiquées par certains Grecs (région que l'on désigne en Grèce sous le nom d'« Epire du Nord » qui correspond au tiers méridional du territoire albanais, au Sud du fleuve Shkumbin). Or ce souvenir de territoires contestés au cours de l'histoire par de groupes nationaux concurrents est demeuré vivace dans les représentations. La présence des migrants albanais a par conséquent été perçue avec une certaine inquiétude par de nombreux Grecs qui pouvaient y voir une menace pour

\footnotetext{
${ }^{6}$ Climat insurrectionnel dans le Sud de l'Albanie en 1997, troubles et intervention militaire au Kosovo en 1999, violences en Macédoine du Nord en 2001
} 
l'intégrité territoriale de la Grèce à une époque où les frontières des pays Balkans semblent mal assurées.

En établissant dès 1998 une différenciation juridique qui octroie un statut préférentiel pour ceux qu'il considère comme des «Grecs d'Albanie », l'État grec s'est fait l'écho de telles représentations dans le traitement administratif des migrants venus d'Albanie, ce qui conduisit à la distinction de jure entre des Albanais " hellénophones » (ou supposés tels) et les autres migrants albanais. D'une façon plus globale, il existe bien en Grèce une loi concernant le droit au séjour des citoyens étrangers d' "origine grecque » (que l'on appelle en Grèce omogéneis) qui date de 1976. Celle-ci a pour conséquences de diviser les étrangers en deux catégories en fonction de leur appartenance à une communauté grecque transnationale existant en dehors des liens juridiques de la citoyenneté (Tsitselikis, 2008). Elle fait de la «nationalité » (ethnikotita), notion mélangeant ascendance, conscience nationale et origine ethnique, un enjeu important dans la détermination de leur statut juridique. Cette migration était également administrée par une institution particulière, la "Fondation pour l'accueil et la réhabilitation des rapatriés grecs» (EIYAPOE) originellement fondée en 1991 pour accueillir des populations d' "origine grecque » venue d'ex-URSS et dont l'implantation avait été programmé par les autorités notamment dans les régions de la minorité turcophone de Grèce (Dallègre 1997).

Il existe donc un ensemble de représentations, hérité du processus de construction de la nation grecque entre le $18^{\mathrm{e}}$ et $20^{\mathrm{e}}$ siècle, qui contribue à structurer les catégories pratiques de la législation migratoire de ce pays en deçà des règlementations communautaires. Ces représentations peuvent également éclairer les pratiques de certains acteurs de la gestion migratoire quotidienne, voire même des logiques de fonctionnement du dispositif frontalier. Dans une telle optique, la frontière doit être vue comme un lieu de contrôle, de filtrage mais donc également de distinction et de sélection au regard de cette somme de critères particuliers.

\section{Le refus d'entrée en Grèce, révélateur de la prévalence des logiques nationales}

Lors du contrôle frontalier, l'individu est théoriquement considéré avant tout en fonction du lien juridique qui l'unit à un État. Les ressortissants de pays extérieurs à l'espace national dans lequel ils souhaitent se rendre sont classés en fonction de l'interaction de la catégorie nationale à laquelle ils appartiennent et du régime de circulation qui y est associé (Jansen, 2009). Ce n'est pourtant pas le cas pour les exemples abordés ci-après où l'individu migrant, en provenance d'un pays voisin de la Grèce, est plutôt ramené à son appartenance supposée à un collectif dont la mobilité transfrontalière est limitée pour des raisons géopolitiques. L'influence des constructions nationales telle que décrites précédemment vient ici ajouter un nouveau niveau de distinction entre les citoyens d'un même pays : certains migrants sont vus comme des indésirables en Grèce, et ils peuvent aller jusqu'à se voir refuser l'entrée sur le territoire, non pas en raison de leur citoyenneté ou de leur situation économique mais de leur appartenance à un sous-groupe dont la présence est jugée dangereuse en Grèce. C'est le cas en particulier des albanophones nés en Grèce avant la seconde guerre mondiale, ceux qu'on appelle les Tchams, qui ont été expulsés du pays en 1945 vers l'Albanie sous le prétexte qu'ils auraient collaboré avec l'ennemi italien et allemand et qu'ils auraient participé à/facilité/commis des crimes en Grèce. Il en va de même des réfugiés 
slavophones qui ont quitté le territoire grec pendant la guerre civile pour se réfugier dans les pays limitrophes ou d'autres républiques du bloc socialiste. Ces différentes personnes sont identifiables par les autorités grecques car leur passeport albanais ou macédonien porte le nom de leur village ou de leur ville de naissance en Grèce avec son toponyme albanais ou slave. C'est à ce titre qu'ils se voient interdire l'accès au territoire grec : ils sont considérés comme des renégats, déchus de leur nationalité grecque, et potentiellement dangereux pour la sécurité nationale car susceptibles de réclamer des biens, des terres, mais aussi de soutenir un discours irrédentiste dans des régions rattachées à la Grèce depuis 1913 (Sintès 2017).

Si la Grèce et ce qui était encore la République de Macédoine ont pu trouver un accord sur cette question 7 , il n'en va pas de même pour l'Albanie. L'entrave mise en Grèce à la circulation des citoyens albanais originaires de Grèce est même une question qui empoisonne régulièrement les relations entre les deux pays depuis plusieurs décennies. En novembre 2016, lors d'une interview à la télévision grecque, le chef de l'État albanais, Edi Rama, ne manquait pas de soulever ce problème tout en étant poussé par le journaliste à se justifier de ne pas masquer ainsi des visées irrédentiste visant à la création d'une "grande Albanie» qui engloberait autant le Kosovo, l'Ouest de la Macédoine du Nord et le Nord-Ouest de la Grèce ${ }^{8}$. Ce type de situation pourrait être vu comme un classique de la géopolitique balkanique, où les conflits autour des questions de minorités ont pu aboutir à l'existence de groupes stigmatisés collectivement par un État et rendu non grata sur son sol. Mais il est intéressant de noter qu'il y a eu au cours des années 2010 une extension surprenante de cette logique nationale au traitement d'autres migrants albanais en Grèce. La presse rapporte en effet que les officiers de police ou de douane grecs présents à la frontière ont à de nombreuses reprises interdit l'accès du pays à de jeunes albanais, nés en Grèce dans les années 1990 et 2000 de parents migrants, car la toponymie choisie sur leur passeport albanais pour désigner leur lieu de naissance en Grèce ne respectait par la nomenclature internationale au profit du terme d'usage dans la langue albanaise (par exemple Selanik pour Thessaloniki ou encore Gumenicë pour Igoumenitsa, Korfuz pour Kerkira), ce choix étant perçu comme un signe d'hostilité voire d'irrédentisme. C'est ainsi qu'à l'été 2013, ce sont des centaines de familles de migrants qui auraient été contraintes de faire marche-arrière à la frontière car le passeport albanais de leur enfant né en Grèce ne portait pas le nom grec de sa ville de naissance?.

\section{Un outil d'intégration paradoxal?}

A la manière de ce que nous venons de montrer concernant la distinction des groupes indésirables, des logiques nationales sont également à l'œuvre quand il s'agit de faire de la frontière un lieu de sélection positive. Le fait de permettre à certains migrants plutôt qu'à d'autres de la franchir témoigne de leur prégnance. Entre l'Albanie et la Grèce, le

\footnotetext{
${ }^{7}$ Les deux pays ont conclu en 1998 un accord permettant aux citoyens macédoniens né en Grèce et ayant quitté le pays durant la guerre civile d'accéder au territoire grec. Cet accord leur offrait la possibilité de ne mentionner que le nom du pays, la Grèce, en tant que lieu de naissance, court-circuitant ainsi la question de la toponymie qui servait de base à la sélection.

${ }^{8} \mathrm{https}$ ///balkaninsight.com/2016/11/23/cameria-becomes-the-focus-of-albanian-pm-interview-in-thegreek-tv-11-23-2016/

${ }^{9} \mathrm{https}$ //www.newsbomb.gr/ellada/story/327065/ellino-alvanika-synora-apagoreysi-eisodoy-sealvanoys
} 
passage peut par exemple se trouver facilité du fait de l'appartenance à la minorité grecque d'Albanie : le statut d'omogéneis, attesté par une carte particulière émise par les consulats grecs d'Albanie, lui attribue une qualité complémentaire à celle de citoyen albanais, en l'identifiant comme un membre de la nation grecque à l'égale des nombreux descendants de la diaspora. Il lui offre ainsi des facilités d'accès, de résidence et de travail sur le territoire grec. Formalisée dans ce cas autour d'un statut administratif, cette position particulière au regard du discours prévalant en Grèce sur l'histoire de la nation peut également relever de processus informels. Même sans accès à l'omogéneia officielle, certains migrants albanais ont ainsi pu bénéficier d'une certaine mansuétude de la part des douaniers ou des policiers quand leur nom de famille ou leur région d'origine laissaient supposer une appartenance à la religion orthodoxe - interprétée comme un facteur favorable à l'émergence d'une véritable "conscience nationale » grecque chez ces migrants d'Albanie (Sintès, 2010).

La présence ou l'absence de dispositif légal ne préjuge pas nécessairement de la prégnance de ces logiques nationales et peut également se révéler être une adaptation à une situation particulière, comme dans le cas des modalités d'accès des citoyens macédoniens au territoire grec. Si, dans la région, l'omogeneia est attribuée aux populations considérées comme grecques en Albanie ou en Bulgarie ${ }^{10}$, les ressortissants de Macédoine du Nord n'ont jamais été concernés par ce dispositif malgré l'importante présence d'une population aroumaine ${ }^{11}$ dans le pays ainsi que la place prise historiquement par certains de ses membres et par certains lieux dans l'imaginaire national grec. La présence aroumaine est néanmoins ici aussi sujette, de façon informelle, à un accès facilité aux documents administratifs régissant l'entrée et le séjour sur le territoire grec pour ces citoyens macédoniens particuliers. Le dispositif administratif présenté pour l'Albanie a juste été ici remplacé par une forme de soustraitance de la sélection des candidats au visa à des associations communautaires aroumaines, proches des autorités consulaires grecques. C'était particulièrement le cas dans le Sud du pays : selon d'anciens responsables de ces associations, elles disposaient d'un jour particulier pour déposer les demandes de visas au bureau consulaire de Skopje et voyaient non-seulement leurs demandes traitées de façon prioritaire mais également avec une bienveillance manifeste, l'obtention d'un visa étant alors quasi-systématique (Javourez, 2017). L'identification des demandeurs comme membres de la communauté aroumaine dépendait donc de l'entremise de ces organisations, alors que les autorités consulaires grecques considéraient comme membres de cette communauté les individus déposant leur dossier par ce biais. Ce fonctionnement n'était de plus pas réservé à la seule question des visas mais également à d'autres dispositifs comme l'attribution de bourses d'études destinées à accueillir des étudiants macédoniens dans les universités grecques. Cette pratique s'appuie sur l'idée que ces Aroumains des pays voisins sont des membres de la communauté hellénique (Sintès, 2008), et vise à atténuer les effets d'une histoire qui les aurait laissés en dehors du territoire de la patrie.

Cette non-application du statut de l'omogeneia aux citoyens macédoniens semble pouvoir s'expliquer par la prise en compte de la situation sur le versant grec de la

\footnotetext{
${ }^{10}$ De nombreuses populations considérées comme grecque ont ainsi eu accès à ce statut au fil du temps, à l'image des communautés grecques d'Égypte et de Méditerranée orientale, de Turquie, d'Albanie, de Bulgarie, et surtout de l'ancienne Union Soviétique, nombreux à s'installer depuis les années 1990 (Tsitselikis, 2008).

${ }^{11}$ Les Aroumains sont des locuteurs d'une langue dialectale proche du roumain, répandue dans le Sud des Balkans, qui concernait traditionnellement des groupes à l'activité pastorale (Gossiaux 2002).
} 
frontière, dans les régions grecques de Macédoine occidentale et centrale où des populations slavophones sont encore présentes malgré les déplacements de populations de la fin de la guerre civile. Leur sort occupe d'ailleurs une place majeure dans le conflit diplomatique qui a opposé la Grèce et la Macédoine du Nord de 1991 à 2019. C'est cette présence qui alimente en effet la peur de la dépossession qu'évoquent certains acteurs grecs et qui motive la position officielle du pays : les slavophones, dont l'intégration à l'État grec est historiquement source d'incertitude, pourraient être un relai en Grèce de discours macédoniens expansionnistes (Skoulariki, 2003). Dans ce contexte, l'entrée en vigueur d'un dispositif officiel reconnaissant la place de certains citoyens macédoniens au sein de la nation grecque compliquerait la donne alors que les pouvoirs publics grecs observent avec méfiance les mouvements identitaires macédoniens qui se développent sur son territoire depuis le début des années 1980.

\section{Un équipement frontalier sur mesure}

Dans le cas de la frontière gréco-albanaise, la sélection positive des omogéneis d'Albanie se traduit de façon très concrète dans le dispositif de surveillance, de contrôle et de passage rencontré le long de la frontière entre les deux Etats. En 2017, celui-ci comportait 6 points de passage entre Grèce et Albanie : 4 points de passage automobile et 2 points de passage pédestre. Cette situation est le résultat d'un processus d'équipement progressif d'une frontière longtemps militarisée et hermétiquement close comme l'atteste les traces encore présentes de loin en loin dans les paysages (un grand nombre de petites casernes aujourd'hui abandonnées destiné à surveiller la zone frontalière, les restes d'anciennes clôtures ou de zones de sécurité ou encore de très nombreux bunkers ou de postes de garde). Dès les années qui ont suivi la chute du rideau de fer, les points de passage pédestre ont été rapidement installés à côté des deux premiers points de passage ouverts aux voitures (Kakavia et Kristalopigi). Il est intéressant de noter que, selon leur degré d'équipement, ces points de passage pour piétons n'ont été ouverts pendant de longues périodes qu'aux seuls frontaliers "d'origine grecque », car leur statut les dispensait de visa et les formalités étaient ainsi bien plus légères. C'était le cas jusqu'en 2004 pour le point frontalier de Sopik, qui n'était du côté albanais qu'un sentier à peine gardé dans les montagnes, alors que s'entassaient côté grec les voitures des personnes qui passaient la frontière à pied. Il en allait de même pour celui de Molivsokepastos, qui a été tout d'abord ouvert uniquement pour les «Grecs d'Albanie » des trois villages frontaliers les plus proches, puis pour tous les migrants en règle (les formalités administratives étant alors assurées par le commissariat de police du village), avant de redevenir à nouveau accessible uniquement aux «Grecs d'Albanie » après l'ouverture à quelques kilomètres à l'est d'un nouveau point de passage routier par lequel les Albanais non titulaires d'une carte d'Omogéneis étaient invités à se présenter. La situation du point de passage d'Ampelonas, au Nordouest de la ville de Ioannina, est comparable à ce dernier, puisque son usage sélectif à l'attention des seuls «Grecs d'Albanie » ne faisait aucun doute pour l'officier en charge de son contrôle en 2010, même s'il avait été équipé par des fonds européens à des fins sans doute très différentes.

Il ne s'agit que d'un point de passage pour piétons, et encore il n'est emprunté que pour les "Epirotes du Nord»; les étrangers et les Grecs ne peuvent absolument pas passer par là. Les Albanais ne peuvent pas entrer non plus car nous n'avons pas de tampon pour viser les passeports.

Entretien avec des agents de police, Ampelonas, 2010 
Il est enfin intéressant de souligner que ce type de point de passage frontalier pédestre a été ouvert par les autorités grecques exclusivement dans les régions où la frontière grecque jouxtait une zone de peuplement hellénophone en Albanie. On pourrait voir dans ce choix, une volonté d'établir une continuité territoriale facilitée avec les villages de la minorité grecque d'Albanie qui n'est pas sans rappeler d'autres situation attestées dans les Balkans, entre Macédoine du Nord et Albanie par exemple, mais surtout entre la Serbie et le Kosovo où la question des circulations « transfrontalières » dans des régions où le tracé frontalier est contesté peut s'incarner dans ces questions très matérielles de l'équipement du dispositif frontalier et de régimes de mobilité pour les habitants.

\section{Conclusion}

Les bouleversements auxquels la Grèce a été confrontée dans les années 1990 et 2000 avec la force des migrations intra-balkaniques et la signature des accords de Schengen ont conduit ce pays à une transformation radicale de son dispositif de contrôle et d'accueil des populations migrantes. Mais si les politiques européennes s'imposent bien au fil de la période dans le cadre législatif, celui-ci demeure influencé par un système de représentations hérité du processus de construction nationale grec qui conduit à la distinction sur le terrain de différents profils de migrants. Alors que les règlementations Schengen établissent les règles du contrôle frontalier entre la Grèce et les citoyens des États voisins, les autorités grecques mettent en vigueur au cours de ces deux décennies de nouvelles catégories fondées sur un positionnement vis-à-vis de l'hellénité plutôt que sur l'unique citoyenneté des intéressés.

Si les régimes de circulation qui prévalent dans la région sont aujourd'hui différents en raison de la dispense de visa de tourisme accordée depuis 2009 et 2010 aux citoyens macédoniens et albanais ${ }^{12}$, la situation n'a pas évolué de façon radicale. Les migrants albanais sont certes beaucoup moins expulsés du territoire grec, mais une simple enquête aux frontières a permis de constater combien leur entrée n'est jamais vraiment facile en raison des très nombreux justificatifs demandés par les services de police et de douane concernant leur capacité à subvenir à leurs besoins comme à leur logement lors de leur séjour en Grèce. De plus, la question des titres de séjours de longue durée comme des visas de travail reste encore sensible. Les dispositifs de sélection positive décrits dans ces quelques pages conservent ainsi toute leur utilité pour les personnes pouvant y prétendre. Mais deux éléments pourraient avoir changé la donne de manière plus notable: l'attractivité de la Grèce au regard de la crise économique que le pays traverse depuis 2008 et le positionnement de ce pays sur les routes migratoires de longue distance, en transit vers des destinations européennes situées plus à l'Ouest. Pour cette dernière raison, la pression migratoire s'est aujourd'hui reportée sur la frontière gréco-turque, où malgré le contexte très internationalisé du dispositif (Pillant, 2017), la tension entre les deux pays reste forte, témoignant de manière plus manifeste encore de la vigueur des logiques nationales ici comme dans l'ensemble de l'UE (Dujmovic et Sintès 2018).

Ainsi, la poursuite du développement de ces processus d'apparence contradictoire (développement des réglementations et des dispositifs à l'échelle européenne et

${ }^{12}$ Cette obligation de visa est levée sous réserve de l’emploi d’un passeport biométrique. 
maintien d'une législation et de pratiques influencées par les représentations locales et les enjeux nationaux) ne peut qu'interroger. Il convient de se demander si ces deux dynamiques ne s'entretiendraient-elles pas mutuellement, à l'image de ce qui a été constaté pour la coopération transfrontalières européenne, où le développement de leur dimension strictement réglementaire semblait permettre également l'expression de certaines configurations locales prégnantes (Javourez, Pillant, Sintès, 2017) ?

\section{Bibliographie}

BROWN, Keith, « From the Balkans to Baghdad (via Baltimore): Labor Migration and the Routes of Empire », Slavic Review, vol. 69, n 4, pp. 816-834 (voir p. 823)

DALLEGRE, Joëlle, «La Thrace grecque - Populations et territoire », collection «Etudes grecques », Paris, L'Harmattan, 1997.

DUJMOVIC, Morgane et SINTES, Pierre, "Chauvinisme frontalier sur la "route des Balkans" », avec M. DUJMOVIC, Hommes et Migrations, 2016, pp. 107-115.

GOSSIAUX Jean-François, Pouvoirs ethniques dans les Balkans, Presses Universitaires de France, Paris, 2002.

GOUNARIS, Basil C., « Emigration from Macedonia in the Early Twentieth Century, » Journal of Modern Greek Studies, vol. 7, no. 1, 1989, pp. 133-53

JANSEN, Stef, "After the Red Passport: Towards an Anthropology of the Everyday Geopolitics of Entrapment in EU's 'Immediate Outside "», Journal of the Royal Anthropological Institute, vol. 15, n 4 , 2009, pp. 815-832

JAVOUREZ, Guillaume, "Permanence et changement aux frontières de l'Europe. Une approche des recompositions frontalières entre Grèce et République de Macédoine », thèse de doctorat, Aix-Marseille Université, 2017

JAVOUREZ, Guillaume, PILLANT, Laurence, SINTES, Pierre, « Les frontières de la Grèce : un laboratoire pour l'Europe? », L’Espace Politique, vol.33, n³, 2017

KONDONIS, Haralambos, « Bilateral Relations Between Greece and the Former Yugoslav Republic of Macedonia ", tiré de KOFOS \& VLASIDIS (éd.), Athens-Skopje : An Uneasy Symbiosis, Thessalonique, ELIAMEP \& Museum of the Macedonian Struggle Foundation, 2005, pp. 55-88

MOROKVASIC, Mirjana, «Une migration pendulaire, les Polonais en Allemagne », Hommes et Migrations $n^{\circ} 1155,1992$, pp. 31-37

PARSANOGLOU, Dimitris, "Grèce, pays d'immigration : Perspectives historiques et sociologiques », thèse de doctorat, EHESS, 2010.

PILLANT, Laurence, «La frontière comme assemblage : géographie crotique du contrôle migratoire à la frontière orientale de la Grèce », thèse de doctorat, Aix-Marseille Université, 2017. 
SINTES Pierre, «En présence du passé : géopolitique de la mémoire aux frontières de la Grèce » collection « sociétés contemporaines », Aix-en-Provence : Presses universitaires de Provence, 2017, 208 p.

SINTES, Pierre, «La raison du mouvement : territoires et réseaux de migrants albanais en Grèce », collection «l'Atelier méditerranéen », Paris : Karthala , 2010, 456 p.

SINTES, Pierre, "Les Valaques de la région de Gjirokastër et la Grèce », dans G. de RAPPER \& P. SINTES (dirs.), Nommer et classer dans les Balkans, collection Mondes Méditerranéens et Balkaniques, EfA, Athènes, 2008, pp. 23-43.

SKOULARIKI, Athena, "La crise macédonienne (1991-1995) et la question des slavophones en Grèce », Balkanologie, vol.7, n¹, 2003, pp. 137-146

de TINGUY, Anne, «Le phénomène des Tchelnoki et la constitution d'espaces transfrontaliers dans la région de la mer Noire», tire de KICHOU (dir.), Méditerranée et Mer Noire entre mondialisation et régionalisation, Paris : L'Harmattan, 2000, 520 p.

TSITSÉLIKIS Konstantinos, " Le droit de la différence: minorités et étrangers en Grèce », tiré de SINTÈS et de RAPPER (dir), Nommer et classer dans les Balkans, Athènes : Ecole Française d'Athènes, 2008, 297 p.

WHITOL de WENDEN Catherine, «Un essai de typologie des nouvelles mobilités », Hommes et Migrations, $n^{\circ} 1233,2000$, pp 5-12 